\title{
SECCIÓN ESPECIAL \\ Reserva de la Biosfera Cabo de Hornos y Parque Marino Islas Diego Ramírez-Paso Drake
}

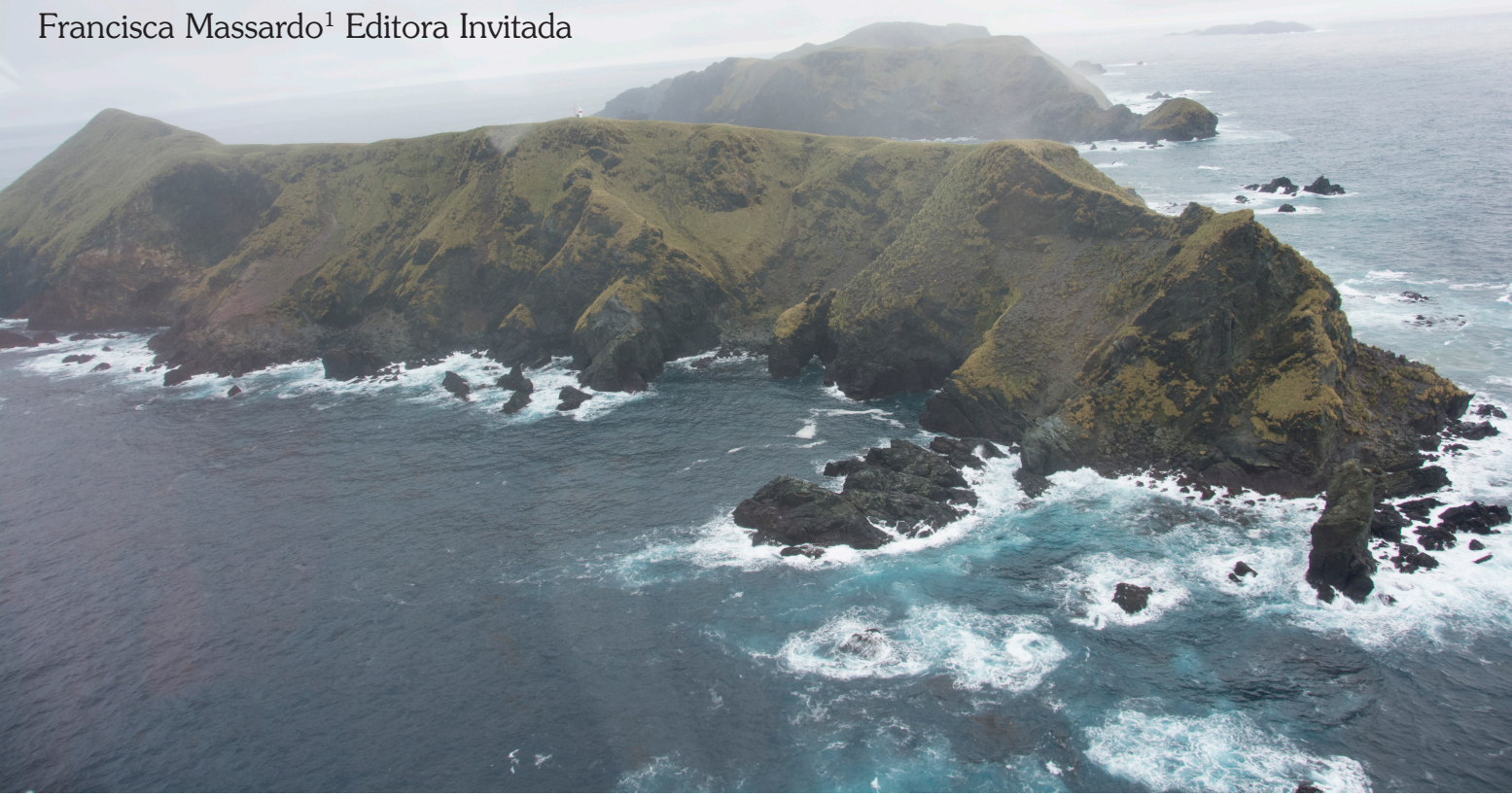

En este número de la revista Anales del Instituto de la Patagonia damos inicio a una sección que hemos llamado "Reserva de la Biosfera Cabo de Hornos y Parque Marino Islas Diego Ramírez-Paso Drake". Investigadores de la Universidad de Magallanes(UMAG), especialmente de la UMAG-Puerto Williams en colaboración con investigadores de otras instituciones nacionales $e$ internacionales, han liderado la preparación de los documentos técnico-científicos para la creación de dos grandes áreas protegidas marino-terrestres en el extremo sur del continente americano. Primero, la Reserva de la Biosfera Cabo de Hornos (RBCH) reconocida por el Programa sobre el Hombre y la Biosfera (MaB, por su nombre en inglés Man and Biosphere) de la Organización de las Naciones Unidas para la Educación, la Ciencia y la Cultura (UNESCO, por su nombre en inglés United Nations Educational, Scientific and Cultural Organization) el año 2005 (Fig. 1). Luego, el Parque Marino Islas Diego Ramírez-Paso Drake (PM Diego Ramírez) cuya creación se oficializó el año 2019 (Fig. 2). La primera protege 4.884.274 de hectáreas (2.967.036 ha marinas y 1.917.238 ha terrestres) y el segundo protege $144.390,6 \mathrm{~km}^{2}$ (Fig. 2).

La creación de estas áreas protegidas ha sido un paso necesario, pero no suficiente, para la conservación de estos ecosistemas únicos a nivel mundial (Rozzi et al. 2007, 2012). Es necesario, además, implementar estas áreas protegidas (UNESCO 1996). Por ello, hace más de veinte años se fundaron el Parque Omora y la Fundación Omora asociados a la UMAG-Puerto Williams como laboratorio natural para desarrollar programas de investigación, educación y conservación biocultural en la $\mathrm{RBCH}$, y más recientemente en el PM Diego Ramírez (Rozzi et al. 2006, 2017).

1 Programa de Conservación Biocultural Subantártica, Universidad de Magallanes, Puerto Williams, Chile, Instituto de Ecología y Biodiversidad.

$\bigotimes$ francisca.massardo@umag.cl;

francisca.massardo@gmail.com

Foto: Vista aérea, desde el sur, de la isla Gonzalo en el archipiélago Diego Ramírez. Detrás se observa la isla Bartolomé. Fotografía de Omar Barroso. 


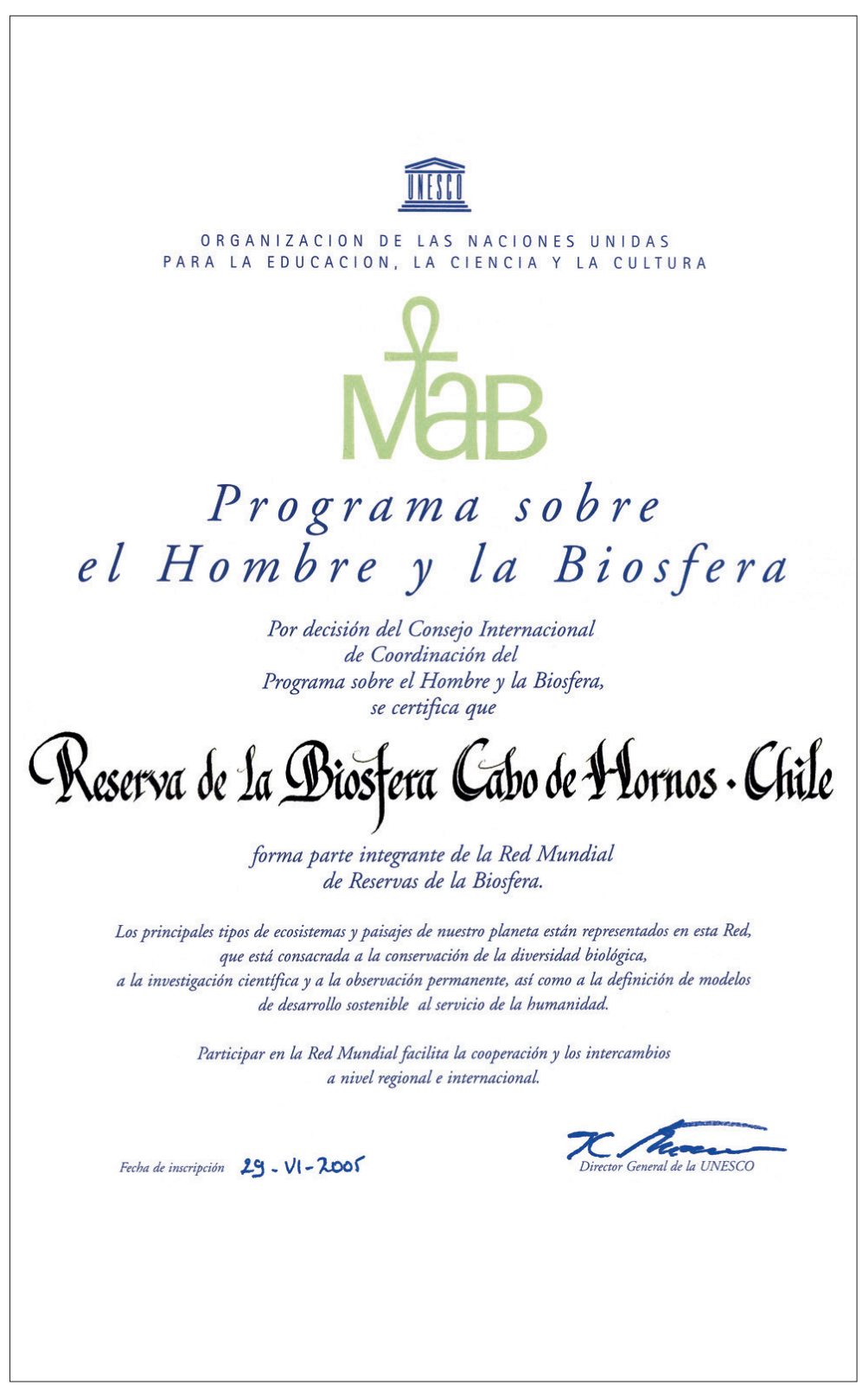

Fig. 1. Certificado de MaB-UNESCO que reconoce la creación de la Reserva de la Biosfera Cabo de Hornos - Chile y su integración a la Red Mundial de Reservas de la Biosfera.

Desde la creación de la $\mathrm{RBCH}$ el año 2005, el equipo científico del Parque Omora estableció el Programa de Conservación Biocultural Subantártica (PCBS), un programa interdisciplinario, interinstitucional e internacional que ha conducido investigación a largo plazo y educación desde el nivel preescolar a la educación superior y formación de capacidades técnicas (Rozzi \& Schüttler, 2015). Las acciones de conservación del PCBS se han basado en la transferencia de conocimiento científico para prácticas económicas sustentables (e.g., turismo de intereses especiales), toma de decisiones ambientales y planificación territorial para la conservación y la sustentabilidad 

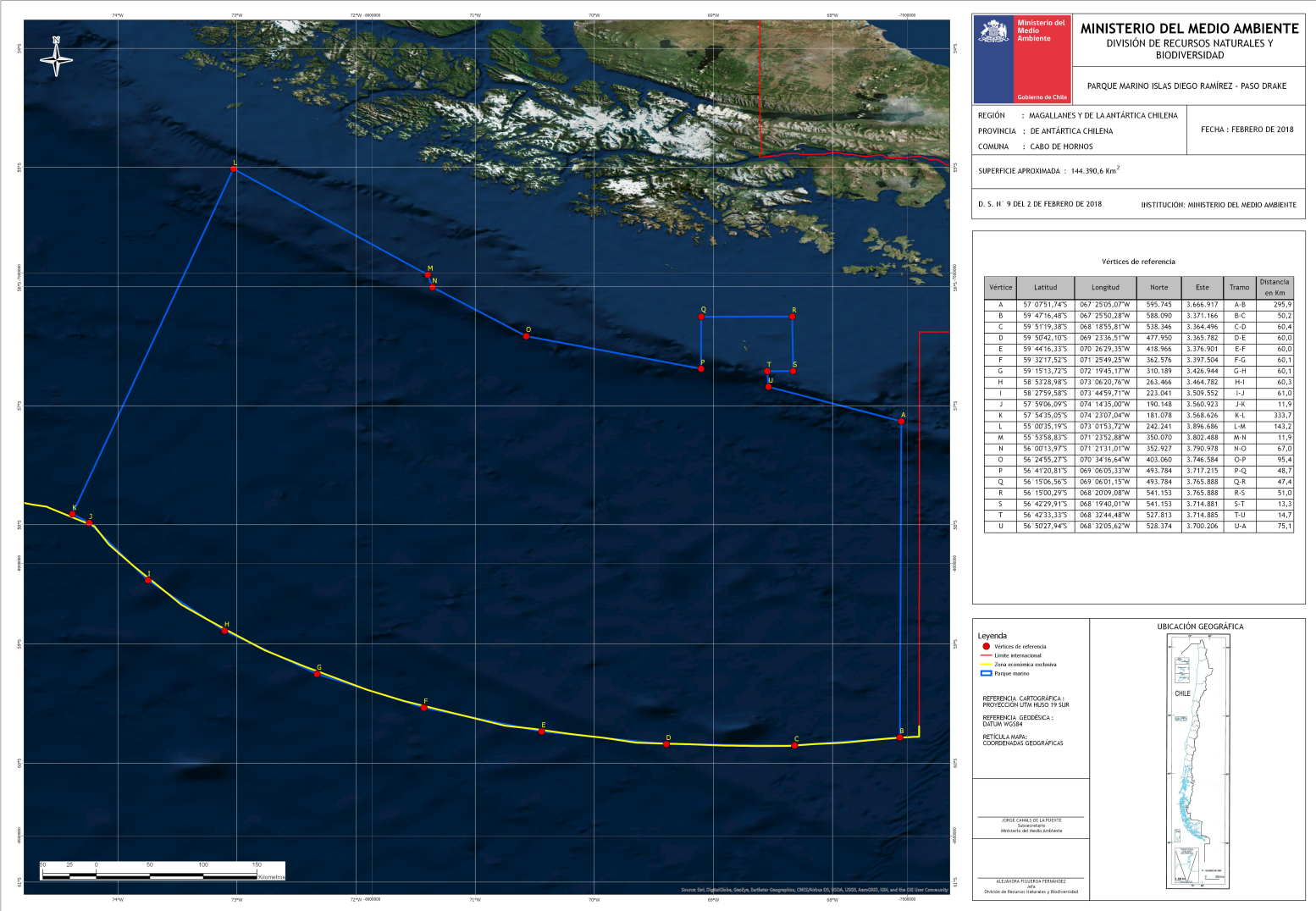

Fig. 2. El Parque Marino Islas Diego Ramírez-Paso Drake se creó el 2 de febrero de 2018, y apareció en el Diario Oficial de la República de Chile del 21 de enero de 2019. Fuente: Ministerio del Medio Ambiente, Chile.

económica, social y ambiental (Schüttler et al. 2011, Mansilla et al. 2012, Contador et al. 2014). Como parte de esta tarea, en febrero del año 2015 el PCBS se comprometió con el Gobierno Regional de Magallanes y Antártica Chilena, el Gobierno de la Provincia Antártica Chilena, la I. Municipalidad de Cabo de Hornos y la Corporación Nacional Forestal (CONAF), a preparar el informe técnicocientífico de la primera década de la $\mathrm{RBCH}$ que serviría de base para la preparación del documento que el Estado de Chile requería para enviar a MaBUNESCO. En septiembre de 2016 este informe se entregó al Gobierno Regional de Magallanes y Antártica Chilena y a la Corporación Nacional Forestal (CONAF) XII Región y Nacional (Massardo et al. 2016). En este informe técnico-científico, Massardo y colaboradores (2016) resumieron las actividades y logros obtenidos en los ámbitos de investigación, educación, capacitación y monitoreo, conservación de la biodiversidad y bases para el desarrollo sustentable, con un énfasis en el turismo de intereses especiales. Se mencionaron también las tres líneas de acción propuestas para la implementación de la $\mathrm{RBCH}$ en su Segunda Década 2016-2025: Centro Subantártico Cabo de Hornos, Red Cabo de Hornos de Estudios Ecológicos a Largo Plazo, y ampliación de la $\mathrm{RBCH}$, incluyendo el archipiélago Diego Ramírez y los ecosistemas marinos hacia el Paso Drake.

En octubre de 2016, el punto focal del Programa MaB-UNESCO y la Cancillería de Chile enviaron el documento "Informe de la Primera Revisión Periódica (2005-2015) de la Reserva de Biosfera Cabo de Hornos" a la oficina central del Programa MaB-UNESCO en París, Francia. Este informe fue aprobado por el Programa MaBUNESCO en marzo de 2017 (Recuadro 1). Esta aprobación representa un hito para Chile, puesto que éste ha sido el primer Informe de Revisión 
Periódica enviado por una reserva de biosfera chilena al Comité $\mathrm{MaB}$ en el plazo exacto de cumplimiento de la primera década.

MaB-UNESCO expresa en la aprobación del Informe de la Primera Revisión Periódica (20052015) de la RBCH la efectividad de los resultados que pueden alcanzarse a través de la alianza ciencia y Estado. Esta Sección Especial que inaugura Anales del Instituto de la Patagonia contribuye significativamente a la implementación de las recomendaciones de MaB-UNESCO en su respuesta al Primer Informe 2005-2015 (Recuadro 1).

En esta primera Sección Especial nos enfocamos en el componente investigación, educación, capacitación y monitoreo de la $\mathrm{RBCH}$ y el PM Diego Ramírez. Un hito reciente ha sido el establecimiento de la Red de Estudios Ecológicos a Largo Plazo de Cabo de Hornos, LTER-Cabo de Hornos (LTER, por el inglés Long-Term Ecological Research). Esta Red LTER-Cabo de Hornos comprende grupos de sitios que investigan la biodiversidad y sus respuestas al cambio global en cuatro sectores clave y contrastantes de la comuna de Cabo de Hornos: (1) Bahía Yendegaia y Canal Beagle, (2) Archipiélago Diego Ramírez, (3) PN Cabo de Hornos en el archipiélago de las islas Wollaston, y (4) isla Navarino (Rozzi et al. en este número).

Los artículos proveen una primera caracterización comprensiva de la composición de especies de la biodiversidad de la isla Gonzalo, en el archipiélago Diego Ramírez, en: (i) ecosistemas costero-marinos, con foco en los ensambles de algas del intermareal (Marambio et al. en este número), $y$ de moluscos (Rosenfeld et al. en este número); (ii) ecosistemas terrestres con foco en las plantas no-vasculares (i.e., briofitas, Goffinet et al. en este número), plantas vasculares (Mackenzie et al. en este número) y aves, considerando por primera vez todas las estaciones del año (Barroso et al. en este número); y (iii) ecosistemas dulceacuícolas, en particular sus invertebrados (Contador et al. en este número). Además, se incluye una primera síntesis de la avifauna de la $\mathrm{RBCH}$ y sus patrones de distribución espacial y temporal (Sandvig et al. en este número).
RECUADRO 1. Respuesta del Programa MaB UNESCO al Informe de la Primera Revisión Periódica de la Reserva de la Biosfera Cabo de Hornos

El 24 de marzo de 2017, el director de la División de Ciencias Ecológicas y de la Tierra del Programa MaBUNESCO envió la respuesta al Primer Informe de Revisión Periódica de la Reserva de la Biosfera Cabo de Hornos. Esta respuesta contiene en el extracto del informe de la 23a reunión del Comité Asesor Internacional para las Reservas de Biosfera realizada el 23-26 de enero de 2017, Sede de la UNESCO, París. En este recuadro incluimos una traducción al español de la sección del extracto de MaB-UNESCO referida a Cabo de Hornos, que señala lo siguiente:

"Reserva de Biosfera Cabo de Hornos (Chile). El Comité Asesor acogió con satisfacción el primer informe de revisión periódica de la Reserva de Biosfera Cabo de Homos, designada en 2005. Este informe de revisión periódica se presentó a la Secretaría del MAB después de la fecha límite del 30 de septiembre de 2016 (se envió una carta a la Secretaría del MAB el 30 de septiembre, explicando el retraso y el 9 de octubre se presentó la Revisión Periódica).

Esta reserva de biosfera forma parte de los bosques siempreverde subpolares (o subantárticos) de Magallanes, y contiene un mosaico de ecosistemas contrastantes con características únicas y singulares.

Se registraron cambios en la configuración espacial de la reserva de biosfera durante 2005-2015. Simultáneamente, los ministerios y servicios involucrados propusieron la ampliación de la reserva de biosfera para proteger áreas terrestres y marinas de gran valor biológico. La conservación a largo plazo de la biodiversidad en este sitio debe abordarse dentro del marco de planificación territorial de la comuna de Cabo de Homos, y debe considerar la integración de áreas terrestres y marinas.

El turismo de bajo impacto se propuso como una estrategia de desarrollo sostenible, de acuerdo con el Plan Estratégico de Desarrollo del gobierno para la región. La coincidencia de límites biogeográficos, administrativos y culturales (relacionados con la distribución ancestral de la etnia originaria) en la reserva de la biosfera permite el desarrollo de formas de turismo que serán económicamente sostenibles a largo plazo.

La Revisión Periódica propone una ampliación significativa de la superficie total de la reserva de biosfera. Anteriormente, el sitio tenía una superficie total de 4.907.657,2 ha. La nueva revisión propone añadir una superficie total de 15.125.611,34 ha, incluidas las islas Diego Ramírez.

El Comité Asesor concluyó que la reserva de biosfera cumple $^{1}$ con los criterios del Marco Estatutario de la Red Mundial de Reservas de Biosfera y señaló que este examen periódico debe considerarse un informe de seguimiento, ya que el próximo examen periódico deberá enviarse en 2019.

Debido al tamaño de la extensión propuesta, el Comité Asesor recomienda a las autoridades chilenas presentar un documento adecuado de esta extensión." 
En los próximos números presentaremos trabajos correspondientes a diversos aspectos de la ecología de las áreas de esta reserva de la biosfera. Esperamos que estos trabajos sean considerados para el futuro manejo las áreas protegidas de la comuna Cabo de Hornos a través del Comité de Gestión de la $\mathrm{RBCH}$. Con esta sección, la UMAG y su revista Anales del Instituto de la Patagonia procuran contribuir a la necesaria colaboración entre la ciencia, la ciudadanía, incluyendo las comunidades locales, las instituciones de educación (pre-escolar, escolar y universitaria), el sector privado y público, particularmente los ministerios y servicios públicos, la Armada de Chile, Carabineros de Chile, los manejadores y tomadores de decisiones y todas aquellas personas interesadas en la conservación del singular patrimonio natural de nuestro país.

\section{AGRADECIMIENTOS}

Agradecemos a la Armada de Chile, Carabineros de Chile, Corporación Nacional Forestal, Dirección General de Aguas del Ministerio de Obras Públicas, Ministerio del Medio Ambiente, Ministerio de Bienes Nacionales, Subsecretaría de Pesca y Subsecretaría de Turismo del Ministerio de Economía, Fomento y Turismo, por el apoyo para la implementación de la Reserva de la Biosfera Cabo de Hornos y/o Parque Marino Islas Diego RamírezPaso Drake. La preparación de este trabajo y de esta Sección Especial que inauguramos en la revista Anales del Instituto de la Patagonia ha contado con el apoyo de los proyectos Bienes PúblicosCORFO a la Universidad de Magallanes (UMAG), Financiamiento Basal ANID (AFB170008) al Instituto de Ecología y Biodiversidad (IEB) y del Grupo de Trabajo Mar y Tierra (The Pew Charitable Trust-Chile) a la Fundación Omora. Esta es una contribución del Programa de Conservación Biocultural Subantártica, conjuntamente coordinado por UMAG, IEB y University of North Texas (UNT).

\section{LITERATURA CITADA}

Barroso, O., Crego, R. D., Mella, J., Rosenfeld, S., Contador, T., Mackenzie, R., Vásquez, R. A., \& Rozzi, R. Colaboración científica con la Armada de Chile en estudios ornitológicos a largo plazo en el archipiélago Diego Ramírez: Primer monitoreo del ciclo anual del ensamble de aves en la isla Gonzalo. Anales del Instituto de la Patagonia, 48(3), 149-168.

Contador, T. A., Kennedy, J. H., Ojeda, J., Feinsinger, P., \& Rozzi, R. (2014). Ciclos de vida de insectos dulceacuícolas y cambio climático global en la ecorregión subantártica de Magallanes: investigaciones ecológicas a largo plazo en el Parque Etnobotánico Omora, Reserva de Biosfera Cabo de Hornos (55 S). Bosque, 35, 429-437.

Contador, T., Rendoll, J., Mackenzie, R., Rosenfeld, S., Barroso, O., Goffinet, B., Kennedy, J., Convey, P. \& Rozzi, R. 2020. Comunidades de invertebrados terrestres del archipiélago Diego Ramírez (56³1's), el sitio de estudios socio-ecológica a largo plazo más austral de América: diversidad y afinidades con las islas subantárticas del Océano Austral. Anales del Instituto de la Patagonia, 48(3), 83-98.

Goffinet, B., Engel, J. J., Von Konrat, M., Mackenzie, R., Contador, T., Rosenfeld, S., Barroso, O., \& Rozzi, R. 2020. First bryophyte records from Diego Ramírez archipelago: changing lenses in long-term socio-ecological research at the southernmost island of the Americas. Anales del Instituto de la Patagonia, 48(3), 127-138.

Mackenzie, R., Vidal, O., Rosenfeld, S., Contador, T., Barroso, O., Goffinet, B., Massardo, F., Arce-Johnson, P., \& Rozzi, R. 2020. Flora vascular y formaciones vegetacionales en el sitio de estudios socio-ecológicos a largo plazo, isla Gonzalo, archipiélago Diego Ramírez (56³1's), Chile. Anales del Instituto de la Patagonia, 48(3), 139-148.

Mansilla, A., Ojeda, J., \& Rozzi, R. 2012. Cambio climático global en el contexto de la ecorregión subantártica de Magallanes y la Reserva de Biosfera Cabo de Hornos. Anales del Instituto de la Patagonia, 40(1), 69-76.

Marambio, J., Rosenfeld, S., Rodríguez, J. P., Méndez, F., Contador, T., Mackenzie, R., Goffinet, B., Rozzi, R., \& Mansilla, A.2020. Siete nuevos registros de macroalgas para 
el archipiélago Diego Ramírez (56³1's): el valor del nuevo parque marino como sumidero de carbono y conservación de la biodiversidad subantártica. Anales del Instituto de la Patagonia, 48(3), 99-111.

Massardo, F., Contador, T., Rendoll-Cárcamo, J., Rosenfeld, Mansilla, A., \& Rozzi, R. (2016) Reserva de la Biosfera Cabo de Hornos (2005-2015). Informe Técnico-Científico para la Primera Revisión Periódica MaBUNESCO. Punta Arenas: Ediciones Universidad de Magallanes.

Rosenfeld, S., Marambio, J., Aldea, C., Rodríguez, J. P., Méndez, F., González-Wevar, C., Gerard, K., Contador, T., Mackenzie, R., Rozzi, R., \& Mansilla, A. 2020. Actualización del catastro de ensamble de moluscos costero-marinos del archipiélago Diego Ramírez (56³1's), chile: un refugio para la economía sustentable y conservación subantártica. Anales del Instituto de la Patagonia, 48(3), 113-125.

Rozzi, R., \& Schüttler, E. (2015). Primera década de investigación y educación en la Reserva de la Biosfera Cabo de Hornos: el enfoque biocultural del Parque Etnobotánico Omora. Anales del Instituto de la Patagonia, 43, 75-99.

Rozzi, R., Massardo, F., Anderson, C. B., Heidinger, K., \& Silander Jr, J. A. (2006a). Ten principles for biocultural conservation at the southern tip of the Americas: the approach of the Omora Ethnobotanical Park. Ecology and Society, 11(1), 43 [online] URL: http:// www.ecologyandsociety.org/vol11/iss1/ art43/

Rozzi, R., Massardo, F., Mansilla, A., Anderson, C. B., Berghöfer, A., Mansilla, M., Gallardo, M. R.,... \& Barros, E. (2007). La reserva de biosfera Cabo de Hornos: un desafío para la conservación de la biodiversidad e implementación del desarrollo sustentable en el extremo austral de América. Anales del Instituto de la Patagonia, 35, 55-70.

Rozzi, R., Armesto, J. J., Gutiérrez, J., Massardo, F., Likens, G., Anderson, C. B., Poole, A., Moses, K., Hargrove, G., Mansilla, A., Kennedy, J. H., Willson, M., Jax, K., Jones, C., Callicott, J. B., \& Kalin, M. T. (2012).
Integrating ecology and environmental ethics: Earth stewardship in the southern end of the Americas. BioScience, 62, 226236.

Rozzi, R., Massardo, F., Mansilla, A., Squeo, F. A., Barros, E., Contador, T., Frangópulos, M., Poulin, E., Rosenfeld, S., Goffinet, B., González-Wevar, C., Mackenzie, R., Crego, R. D., Viddi, F., Naretto, J., Gallardo, M. R., Jiménez, J. E., Marambio, J.,... \& Martinic, M. (2017). Parque Marino Cabo de Hornos-Diego Ramírez. Technical report to the proposal for creation of the Cape Horn - Diego Ramirez Marine Park. Punta Arenas: Ediciones Universidad de Magallanes. https://issuu.com/umag9/ docs/ebook_low_parque_marino_umag_ fin_di

Rozzi, R., Crego, R. D., Contador, T., Schüttler, E., Rosenfeld, S., Mackenzie, R., Barroso, O., Silva-Rodríguez, E. A., Álvarez-Bustos, X., Silva, A., Ramírez, I., Mella, J., Herreros, J., Rendoll-Cárcamo, J., Marambio, J., Ojeda, J., Méndez, F., Moses, K. P.,... \& Massardo, F. (2020). Un centinela para el monitoreo del cambio climático y su impacto sobre la biodiversidad en la cumbre austral de américa: la nueva red de estudios ecológicos a largo plazo Cabo de Hornos. Anales del Instituto de la Patagonia, 48(3), 45-82.

Sandvig, E. M., Quilodrán, C. S., Aguirre, F., Rivero de Aguilar, J., Barroso, O., Vásquez, R. A., \& Rozzi, R. 2020. Patrones de distribución de la avifauna de los bosques de la Reserva de la Biosfera Cabo de Hornos: Un antecedente básico para la planificación del aviturismo sustentable. Anales del Instituto de la Patagonia, 48(3), 169-183.

Schüttler, E., Rozzi, R., \& Jax, K. (2011). Towards a societal discourse on invasive species management: a case study of public perceptions of mink and beavers in Cape Horn. Journal for Nature Conservation, 19, 175-184.

UNESCO. (1996). Reservas de biosfera: la estrategia de Sevilla y el marco estatutario de la red mundial. París: UNESCO. 